VORWORT

FOREWORD

006 THILO FOLKERTS

BILDWIRKLICHKEITEN

\title{
FAKE REALITIES
}

009 MARTIN REIN-CANO UND LORENZ DEXLER IM GESPRÄCH MIT THILO FOLKERTS

MARTIN REIN-CANO AND LORENZ DEXLER IN CONVERSATION WITH THILO FOLKERTS

GEGENSTÄNDE AUF EINER PLATTFORM

OBJECTS ON A PLATFORM

055

MARC TREIB

AUF EINER FALSCHEN KUH ÜBER EINE BEWEGTE LANDSCHAFT ZUM STRAND REITEN RIDING A FAKE COW ON A MOVABLE LANDSCAPE, HEADING TO THE BEACH

PIETRO VALLE

DIE KÜNSTLICHKEIT DER STADTNATUR

THE ARTIFICIALITY OF URBAN NATURE

123

BRIGITTE FRANZEN

DAS BAHNDECKEL PROJEKT - ORT, ENTWICKLUNG, TECHNISCHE DETAILS

THE BAHNDECKEL PROJECT - SITE, DEVELOPMENT, TECHNICAL DETAILS

157

LORENZ DEXLER MIT/ WITH THILO FOLKERTS

BIOGRAFIEN

241 BIOGRAPHIES

PROJEKTBETEILIGTE

244 PROJECT CONTRIBUTORS

BILDNACHWEIS

245 ILLUSTRATION CREDITS 